OPEN ACCESS

Edited by:

Yilong Wang,

Capital Medical University, China

Reviewed by:

Jahnavi Kedare,

T. N. Medical Medical College, India

Sara L. Weisenbach,

Stony Brook Medicine, United States

*Correspondence:

Yonghua Huang

huangyonghua2017@126.com

Specialty section:

This article was submitted to

Aging Psychiatry,

a section of the journal

Frontiers in Psychiatry

Received: 31 March 2021

Accepted: 08 July 2021

Published: 03 August 2021

Citation:

Zhào H, Liu Y, Xia Z, Xie H and Huang $Y$ (2021) Diagnosis and Assessment of Apathy in Elderly Chinese Patients With Cerebral Small Vessel Disease.

Front. Psychiatry 12:688685 doi: $10.3389 /$ fpsyt.2021.688685

\section{Diagnosis and Assessment of Apathy in Elderly Chinese Patients With Cerebral Small Vessel Disease}

\author{
Hóngyi Zhào ${ }^{1,2}$, Yu Liu ${ }^{3}$, Zhenxi Xia ${ }^{1}$, Hongyang Xie ${ }^{1}$ and Yonghua Huang ${ }^{1 *}$ \\ ${ }^{1}$ Department of Neurology, Seventh Medical Center of Chinese PLA General Hospital, Beijing, China, ${ }^{2}$ Department of \\ Psychiatry, NO 984 Hospital of People's Liberation Army, Beijing, China, ${ }^{3}$ Department of Neurology, NO 984 Hospital of \\ People's Liberation Army, Beijing, China
}

Objective: The study aimed to estimate the frequency of apathy in Chinese patients with cerebral small vessel disease (CSVD) and investigate the relationship between apathy and neuroimaging markers of CSVD.

Methods: A total of 150 CSVD aged patients were recruited for a cross-sectional observational study. Following the new revised version of diagnostic criteria for apathy (DCA), each patient was evaluated successively by the neuropsychiatric inventory (NPI-apathy), geriatric depression scale (GDS), and caregiver burden scale (CBS). The MRI presence of lacunes, white matter hyperintensities, cerebral microbleeds, and perivascular spaces were rated independently. Furthermore, presence of all these MRI markers were summed in a score of 0-4 representing all CSVD features combined.

Results: According to the DCA, we found that the frequency of apathy in Chinese Alzheimer's disease patients reached $37.33 \%$, with lack of and diminished goal-directed activities in the dimension of behavior/cognition. We did not find a close relationship between apathy and depression. Caregiver burden was positively correlated with apathy severity. Apathy, but not depression, was positively associated with total CSVD burden, rather than a separate MRI marker of CSVD.

Conclusion: As a key component of neuropsychiatric symptoms, apathy was common in Chinese elderly with CSVD, more attention should be paid to apathy in clinical practice of CSVD.

\section{Keywords: cerebral small vessel disease, apathy, neuropsychiatric disorder, aging, depression}

\section{INTRODUCTION}

Cerebral small vessel disease (CSVD) refers to a group of pathological processes which affect the small arteries, arterioles, venules, and capillaries of the brain (1). The features of CSVD range from asymptomatic radiological markers occurrence to symptomatic characteristics including cognitive deficits and gait decline (2). Recent studies reported that neuropsychiatric symptoms (NPS) are quite common in CSVD patients (3-5).

In major contributions, apathy was defined as a lack of motivation that persists over time and causes identifiable functional impairment (6). It has been confirmed that apathy can be found in many neurological diseases, such as Alzheimer's disease (AD), Parkinson disease (PD), Huntington disease (HD), etc., (7-9). Based on the vast sub-cortical changes in CSVD patients that overlap with undermined brain circuits in apathy (10), vascular apathy was hypothesized recently (11). 
Tay et al. (12) proposed that apathy found in CSVD patients might be a disconnection syndrome, which was driven by disruption of white matter tracts connecting regions important for motivation. In detail, the connections between frontalsubcortical circuits, including the anterior cingulate cortex, might be responsible for the initiation of goal-directed behaviors (13). Furthermore, it is reported that the presence of apathy significantly affects the patient's quality of life (14), and the severity of apathy is associated with a faster cognitive and functional decline in neurodegenerative diseases (15).

However, apathy is under-recognized and has been poorly understood in cerebrovascular disease clinical practice for a long time, although, the occurrence of lack of motivation reached onethird in patients after stroke (16). Apathy was easily neglected, even misconstrued as depression among post-stroke patients (17). In the last decade, along with the considerable advances in the domain of apathy in brain disorders, including the apathy biological- and neural-based, the consensus of diagnosis and assessment of apathy has been revised several times $(6,18)$. According to the latest version of diagnostic criteria of apathy (DCA) in brain disorders proposed in 2018 (Nice, France), the spectrum of apathy was enlarged into three dimensions: lack of or diminished goal-directed behavior-cognition, emotion, and social interaction. The purpose of the present study was to investigate apathy and the relevant phenotype in elderly Chinese patients with CSVD, and to investigate the relationship between apathy severity and cognitive disorders in aged CSVD patients.

\section{METHODS}

\section{Participants}

We conducted a clinical cross-sectional observational study from January 1, 2019 to April 1, 2020 and recruited 150 elderly patients with CSVD consecutively from the Department of Neurology at the Seventh Medical Center of PLA General Hospital (Beijing, China). Our study was approved by the Academic Ethics Committee of the Biological Sciences Division of PLA General Hospital in Beijing, China.

The exclusion criteria were: patients with major stroke or cerebral bleeding episodes; other causes of leukoencephalopathy (e.g., immune, demyelination, genetic); major psychiatric diseases; use of psychotropic medications; multisystem diseases such as polyarteritis nodosa, nervous system vasculitis associated with connective tissue disorders, vasculitis secondary to infectious, etc.; arthritis; MRI contraindications; and neurodegenerative dementia.

\section{Magnetic Resonance Imaging Measurements}

A 3.0T MRI brain (Discovery MR750; GE Healthcare, USA) scan displayed white matter lesions reflecting the degree of SVD. A brain MRI (slice and interslice thicknesses of 5 and $1.5 \mathrm{~mm}$, respectively) was carried out as follows: T1 fluidattenuated inversion recovery (TR, $1750 \mathrm{~ms}$; TE, $23 \mathrm{~ms}$; T1, $780 \mathrm{~ms}$; FOV, $24 \mathrm{~cm}$ ) and T2-weighted imaging (TR, $7498 \mathrm{~ms}$; TE, $105 \mathrm{~ms}$; FOV, $24 \mathrm{~cm}$ ) sequences. The assessors were blinded to imaging findings.

\section{Total CSVD Burden Score}

The total CSVD burden score was calculated according to Chen et al. (19). Briefly, one point was allocated to each of the following MRI parameters: severe WMH (periventricular WMH Fazekas 3 or deep WMH Fazekas 2-3), presence of lacunes, microbleeds, and moderate to severe BG-PVS (semi-quantitative rating $>1$ ), with total scores ranging from 0 to 4 points.

\section{Diagnosis and Assessment of Apathy}

Each patient was diagnosed according to the DCA (6) (shown in Supplementary Material 1), and assessed by NPI-apathy, geriatric depression scale (GDS), mini-mental evaluation scale (MMSE), and care-giver burden scale (CBS). Information was collected from caregivers if necessary. All assessments were conducted according to the Chinese version of the above guidelines, and data were organized and completed in the following $24 \mathrm{~h}$.

\section{Statistical Analysis}

Differences between the groups' clinical and demographic data were analyzed by using one-way analysis of variance. Bivariate correlation was selected to detect the correlation between CBS and neuropsychiatric disorders, as well as the relationship between severity of apathy and depression. Stepwise multiple linear regression was used to investigate the correlation between apathy/depression severity and CSVD biomarkers, controlling for age, sex, education, and MMSE score. The significance threshold was set at $P<0.05$ in all statistical tests. Analysis was carried out using SPSS 22.0 software.

\section{RESULTS}

According to the DCA, the frequency of apathy in Chinese AD patients was $37.33 \%$. Table 1 demonstrates the demographic characteristics of our subjects. The apathetic group scored 21.05 \pm 4.09 in MMSE, compared to $24.58 \pm 4.06$ in the non-apathetic group. There were significant differences in the scores between apathetic and non-apathetic groups $(P=0.000)$. Meanwhile, apathetic group patients showed more obvious CSVD burden severity relative to non-apathetic group individuals (CSVD burden score: $2.09 \pm 1.00$ vs. $1.65 \pm 0.84, P=0.004$ ).

Furthermore, according to criterion B of DCA, most Chinese apathetic elderly CSVD patients exhibited a lack of or diminished goal-directed activities in dimension behavior/cognition (78.57\%), followed by goal-directed social interaction (64.28\%), and goal-directed emotion (64.28\%). Details are listed in Table 2.

The severity of depression was evaluated by GDS, and distance correlation of GDS score and NPI-apathy was only $0.158(P$ $=0.054$ ) which indicated a low correlation between apathy and depression.

Caregiver burden was indicated by CBS. The correlation coefficient between CBS score and NPI-apathy, MMSE, and GDS score was $0.485,-0.319$, and 0.300 , respectively. This result indicated that caregiver burden was most closely correlated with apathy severity in aged CSVD patients.

Lastly, stepwise multiple linear regression was adopted to analyze the relationship between apathy severity and MRI 
TABLE 1 | Clinical and demographic characteristics of the subjects with and without apathy.

\begin{tabular}{|c|c|c|c|c|}
\hline & Overall $(N=150)$ & Apathy $+(N=56)$ & Apathy- $(N=94)$ & $P$-value \\
\hline Men, \% & 79 (52.66\%) & 27 (48.21\%) & 52 (55.32\%) & 0.399 \\
\hline Age, years & 70.59 (8.02) & $72.00(8.57)$ & $65.76(7.59)$ & 0.097 \\
\hline Education, years & $8.49(3.47)$ & $8.11(3.71)$ & 8.72 (3.32) & 0.294 \\
\hline NPI-apathy & $3.86(4.05)$ & $6.68(3.45)$ & $2.18(3.40)$ & $0.000^{\#}$ \\
\hline MMSE score & $23.43(4.32)$ & $21.05(4.09)$ & $24.58(4.06)$ & $0.000^{\#}$ \\
\hline CSVD burden score & $1.81(0.92)$ & $2.09(1.00)$ & $1.65(0.84)$ & $0.004^{\#}$ \\
\hline Fazekas score & $1.95(0.91)$ & $2.13(0.83)$ & $1.84(0.94)$ & 0.056 \\
\hline
\end{tabular}

Mean (standard deviation). MMSE, Mini-mental state evaluation; CSVD, Cerebral small vessel disease.

${ }^{\#} P<0.05$ apathy+ relative to apathy-

TABLE 2 | Presence of CSVD aged patients in each apathetic dimension according to DCA.

\begin{tabular}{lcccc}
\hline Dimension & $\boldsymbol{N}$ & B1-behavior/cognition $\boldsymbol{N}(\%)$ & B2-emotion $\boldsymbol{N}$ (\%) & B3-social interaction $\boldsymbol{N}$ (\%) \\
\hline Apathy+ & 56 & $44(78.57 \%)$ & $29(51.78 \%)$ & $36(64.28 \%)$ \\
Apathy- & 94 & $10(10.64 \%)$ & $7(7.45 \%)$ & $5(5.32 \%)$ \\
\hline
\end{tabular}

markers of CSVD such as WMH, LI number, CMB number, BGPVS score, and total CSVD burden score, controlling for age, sex, education, and MMSE score. We only found that NPI-apathy score was closely associated with total CSVD burden $\left(\mathrm{R}^{2}=0.332\right.$, $\mathrm{t}=4.134, P<0.000)$. Whereas, GDS score was not significantly associated with total CSVD burden $\left(\mathrm{R}^{2}=0.036, \mathrm{t}=0.364, P=\right.$ 0.674) and other specific CSVD MRI markers.

\section{DISCUSSION}

Apathy has gained more and more attention in recent years as one of the most common neuropsychiatric symptoms associated with CSVD (20), although, the prevalence of apathy has not reached a consensus. The prevalence of apathy in the general population ( $\geq 50$ years) was estimated at $23.7 \%$ (21). Diagnosed based on neuropsychiatric inventory assessment, Reyes et al. (22) found that apathy frequency was $41 \%$ in cerebral autosomal dominant arteriopathy with subcortical infarcts and leukoencephalopathy (CADASIL) patients (a genetic model of CSVD). In the current study, we revealed that the prevalence of apathy was $37.33 \%$ in Chinese aged patients with sporadic CSVD. These distinct findings of apathy frequency might be caused by the choices of diagnostic methods. Owing to the revision of DCA, more consistency studies of apathy in neuropsychiatric disorders can emerge in future. As has been mentioned by Robert et al. (6), goal-directed activities classified in criterion B of DCA were changed from domains of "behavior, cognition, and emotion" to dimensions of "behavior/cognition, emotion, and social interaction." According to the renewed criterion B of DCA, most Chinese apathetic elderly CSVD patients exhibited loss of or diminished goal-directed activities in dimension behavior/cognition, followed by goal-directed social interaction, and goal-directed emotion.
Depression is another major neuropsychiatric symptom in cerebrovascular disease (3). As far as we know, there is plenty of literature that has found a relationship between depression and CSVD. Vascular depression was proposed by Alexopoulos (23) even earlier than vascular apathy. CSVD patients showed depressive symptoms if the subcortical limbic structure was involved (24). Also, vascular depression was reported to be associated with "depression-executive dysfunction syndrome" (25). However, apathy and depression are dissociable, in spite of the existence of several overlapping features, such as loss of pleasure and reduced energy (16). Apathy and depression should be differentiated in clinical practice, since apathy, but not depression, was associated with executive dysfunction in CSVD (26). Similar findings were also detected in AD patients in our previous study (7). In our current study, we only found an average GDS score of $6.56 \pm 4.60$, which implied that depressive symptoms were not severe in aged CSVD patients. Meanwhile, GDS score was not significantly associated with total CSVD burden or other specific CSVD MRI markers. These findings were not similar to the conclusions of Pasi et al. (27) and Direk et al. (28), which supported a vascular depression hypothesis in CSVD. The main explanation might be that the subjects we recruited were inpatients with more severe cognitive deficits (MMSE: $23.43 \pm 4.32$ ). As has been mentioned by Mortby et al. (24), "apathy become[s] universal among the severely cognitively impaired, depression is an initial symptom, already apparent in the pre-clinical stage of MCI."

Actually, investigators have confirmed that the underlying mechanisms of apathy and depression in CSVD are distinct. For example, Eurelings et al. (29) concluded that increased C-reactive protein levels (a low-grade inflammation) were associated with apathy symptoms but not with depressive symptoms. At the same 
time, Hollocks et al. (13) managed to revealed that white matter microstructural changes in small vessel disease are associated with apathy but not directly with depressive symptoms.

In the present study, we did not detect a significant association between apathy severity and MRI markers of CSVD, except CSVD burden score. The definition of "total CSVD score" was developed by the Maastricht collaborative group to estimate CSVD burden by summing up the presence of lacunes, white matter hyperintensities, cerebral microbleeds, and perivascular spaces independently into an ordinal score (30). As we know, these MRI markers of CSVD do not occur separately, and Wardlaw et al. (31) suggested searching for methods to assess the total CSVD load on imaging in order to avoid over-reliance on one feature only. This might explain why we did not find a statistically close relationship between apathy severity and separate MRI markers of CSVD.

We also found that apathetic group individuals exhibited a more obvious cognitive decline in comparison with the nonapathetic group. In addition, caregiver burden of apathetic group patients was markedly higher than non-apathetic group subjects. These results were consistent with many other reports that have demonstrated that the severity of apathy correlates with caregiver burden in patients with $\mathrm{AD}$, frontotemporal dementia, amyotrophic lateral sclerosis, etc., (7, 32-34). The increase of caregiver burden might result from a decrease in physical activities and daily living ability popularly found in apathetic patients (32). These findings implied that recognizing, diagnosing, and assessing apathy properly is helpful to alleviate apathy-associated caregiver burden.

Several limitations of this study should be considered. First, the sample size was small. Second, there is emerging evidence that new information and communication technology approaches are capable of providing clinicians with valuable additional information in terms of assessment, and therefore, more accurate diagnosis of apathy including actigraphy devices is possible (35). Whereas, we did not select an objective method to assess apathy in our investigation. In a future study, we will collect more information around apathy.

In conclusion, the prevalence of apathy was quite high in elderly Chinese patients with CSVD. Based on MRI imaging, apathetic patients had a more severe SVD burden. Severity of

\section{REFERENCES}

1. Pantoni L. Cerebral small vessel disease: from pathogenesis and clinical characteristics to therapeutic challenges. Lancet Neurol. (2010) 9:689701. doi: 10.1016/S1474-4422(10)70104-6

2. Chen X, Wang J, Shan Y, Cai W, Liu S, Hu M, et al. Cerebral small vessel disease: neuroimaging markers and clinical implication. J Neurol. (2019) 266:2347-62. doi: 10.1007/s00415-018-9077-3

3. Xu X, Chan QL, Hilal S, Goh WK, Ikram MK, Wong TY, et al. Cerebral microbleeds and neuropsychiatric symptoms in an elderly Asian cohort. $J$ Neurol Neurosurg Psychiatry. (2017) 88:7-11. doi: 10.1136/jnnp-2016-313271

4. Baker JG, Williams AJ, Ionita CC, Lee-Kwen P, Ching M, Miletich RS. Cerebral small vessel disease: cognition, mood, daily functioning, and imaging findings from a small pilot sample. Dement Geriatr Cogn Dis Extra. (2012) 2:169-79. doi: $10.1159 / 000333482$ apathy, rather than depression, was positively associated with CSVD burden. In CSVD aged patients, caregiver burden was closely correlated with apathy severity.

\section{DATA AVAILABILITY STATEMENT}

The original contributions presented in the study are included in the article/Supplementary Material, further inquiries can be directed to the corresponding author/s.

\section{ETHICS STATEMENT}

Our study was approved by the Academic Ethic Committee of the Biological Sciences Division of Seventh Medical Center of Chinese PLA General Hospital in Beijing, China. The patients/participants provided their written informed consent to participate in this study.

\section{AUTHOR CONTRIBUTIONS}

$\mathrm{HZ}$ and ZX were responsible for data collection. YL was responsible for manuscript writing. HX was responsible for data analysis. YH was responsible for the study design. All authors contributed to the article and approved the submitted version.

\section{FUNDING}

This study was supported by the Wu Jieping Foundation (Grant No.: 320.6750.18456).

\section{ACKNOWLEDGMENTS}

We thank Dr. Shaopeng Wang for their support in data collection.

\section{SUPPLEMENTARY MATERIAL}

The Supplementary Material for this article can be found online at: https://www.frontiersin.org/articles/10.3389/fpsyt. 2021.688685/full\#supplementary-material
5. Kim HJ, Kang SJ, Kim C, Kim GH, Jeon S, Lee JM, et al. The effects of small vessel disease and amyloid burden on neuropsychiatric symptoms: a study among patients with subcortical vascular cognitive impairments. Neurobiol Aging. (2013) 34:1913-20. doi: 10.1016/j.neurobiolaging.2013.01.002

6. Robert P, Lanctôt KL, Agüera-Ortiz L, Aalten P, Bremond F, Defrancesco $\mathrm{M}$, et al. Is it time to revise the diagnostic criteria for apathy in brain disorders? The 2018 international consensus group. Eur Psychiatry. (2018) 54:71-6. doi: 10.1016/j.eurpsy.2018.07.008

7. Zhao H, Zhao Z, Huang L, Preter M. Diagnosis and assessment of apathy in Chinese patients with Alzheimer's disease. $J$ Psychosom Res. (2012) 72:405-7. doi: 10.1016/j.jpsychores.2012.0 1.003

8. Brown DS, Barrett MJ, Flanigan JL, Sperling SA. Clinical and demographic correlates of apathy in Parkinson's disease. J Neurol. (2019) 266:50714. doi: $10.1007 /$ s00415-018-9166-3 
9. Fritz NE, Boileau NR, Stout JC, Ready R, Perlmutter JS, Paulsen JS, et al. Relationships among apathy, health-related quality of life, and function in Huntington's disease. J Neuropsychiatry Clin Neurosci. (2018) 30:194201. doi: 10.1176/appi.neuropsych.17080173

10. Guimarães HC, Levy R, Teixeira AL, Beato RG, Caramelli P. Neurobiology of apathy in Alzheimer's disease. Arq Neuropsiquiatr. (2008) 66:43643. doi: 10.1590/S0004-282X2008000300035

11. Wouts L, van Kessel M, Beekman ATF, Marijnissen RM, Oude Voshaar RC. Empirical support for the vascular apathy hypothesis: a structured review. Int J Geriatr Psychiatry. (2020) 35:3-11. doi: 10.1002/gps.5217

12. Tay J, Tuladhar AM, Hollocks MJ, Brookes RL, Tozer DJ, Barrick TR, et al. Apathy is associated with large-scale white matter network disruption in small vessel disease. Neurology. (2019) 92:e1157-67. doi: 10.1212/WNL.0000000000007095

13. Hollocks MJ, Lawrence AJ, Brookes RL, Barrick TR, Morris RG, Husain M, et al. Differential relationships between apathy and depression with white matter microstructural changes and functional outcomes. Brain. (2015) 138:380315. doi: 10.1093/brain/awv304

14. Yeager CA, Hyer L. Apathy in dementia: relations with depression, functional competence, and quality of life. Psychol Rep. (2008) 102:71822. doi: $10.2466 /$ pr0.102.3.718-722

15. Starkstein SE, Jorge R, Mizrahi R, Robinson RG. A prospective longitudinal study of apathy in Alzheimer's disease. J Neurol Neurosurg Psychiatry. (2006) 77:8-11. doi: 10.1136/jnnp.2005.069575

16. Tay J, Morris RG, Markus HS. Apathy after stroke: diagnosis, mechanisms, consequences, and treatment. Int J Stroke. (2021) 16:510-8. doi: 10.1177/1747493021990906

17. Sachdev PS. Post-stroke cognitive impairment, depression and apathy: untangling the relationship. Am J Geriatr Psychiatry. (2018) 26:3013. doi: 10.1016/j.jagp.2017.12.002

18. Robert P, Onyike CU, Leentjens AF, Dujardin K, Aalten P, Starkstein $\mathrm{S}$, et al. Proposed diagnostic criteria for apathy in Alzheimer's disease and other neuropsychiatric disorders. Eur Psychiatry. (2009) 24:98104. doi: 10.1016/j.eurpsy.2008.09.001

19. Chen H, Wan H, Zhang M, Liu G, Wang X, Wang Z, et al. Cerebral small vessel disease may worsen motor function, cognition, and mood in Parkinson's disease. Parkinsonism Relat Disord. (2021) 83:86-92. doi: 10.1016/j.parkreldis.2020.12.025

20. Clancy U, Gilmartin D, Jochems ACC, Knox L, Doubal FN, Wardlaw JM. Neuropsychiatric symptoms associated with cerebral small vessel disease: a systematic review and meta-analysis. Lancet Psychiatry. (2021) 8:22536. doi: 10.1016/S2215-0366(20)30431-4

21. Yao H, Takashima Y, Araki Y, Uchino A, Yuzuriha T, Hashimoto M. Leisuretime physical inactivity associated with vascular depression or apathy in community-dwelling elderly subjects: The Sefuri study. J Stroke Cerebrovasc Dis. (2015) 24:2625-31. doi: 10.1016/j.jstrokecerebrovasdis.2015.07.018

22. Reyes S, Viswanathan A, Godin O, Dufouil C, Benisty S, Hernandez K, et al. Apathy: a major symptom in CADASIL. Neurology. (2009) 72:90510. doi: 10.1212/01.wnl.0000344166.03470.f8

23. Alexopoulos GS. The vascular depression hypothesis: 10 years later. Biol Psychiatry. (2006) 60:1304-5. doi: 10.1016/j.biopsych.2006.09.006

24. Mortby ME, Maercker A, Forstmeier S. Apathy: a separate syndrome from depression in dementia? A critical review. Aging Clin Exp Res. (2012) 24:30516. doi: $10.3275 / 8105$

25. Alexopoulos GS. "The depression-executive dysfunction syndrome of late life": a specific target for D3 agonists? Am J Geriatr Psychiatry. (2001) 9:229. doi: 10.1097/00019442-200102000-00004
26. Lohner V, Brookes RL, Hollocks MJ, Morris RG, Markus HS. Apathy, but not depression, is associated with executive dysfunction in cerebral small vessel disease. PLoS ONE. (2017) 12:e0176943. doi: 10.1371/journal.pone.017 6943

27. Pasi M, Poggesi A, Salvadori E, Diciotti S, Ciolli L, Del Bene A, et al. White matter microstructural damage and depressive symptoms in patients with mild cognitive impairment and cerebral small vessel disease: the VMCITuscany study. Int J Geriatr Psychiatry. (2016) 31:611-8. doi: 10.1002/gps. 4368

28. Direk N, Perez HS, Akoudad S, Verhaaren BF, Niessen WJ, Hofman A, et al. Markers of cerebral small vessel disease and severity of depression in the general population. Psychiatry Res Neuroimaging. (2016) 253:1-6. doi: 10.1016/j.pscychresns.2016.0 5.002

29. Eurelings LS, Richard E, Eikelenboom P, van Gool WA, Moll van Charante EP. Low-grade inflammation differentiates between symptoms of apathy and depression in community-dwelling older individuals. Int Psychogeriatr. (2015) 27:639-47. doi: 10.1017/S1041610214002683

30. Staals J, Makin SD, Doubal FN, Dennis MS, Wardlaw JM. Stroke subtype, vascular risk factors, and total MRI brain small-vessel disease burden. Neurology. (2014) 83:1228-34. doi: 10.1212/WNL.0000000000000837

31. Wardlaw JM, Smith C, Dichgans M. Mechanisms of sporadic cerebral small vessel disease: insights from neuroimaging. Lancet Neurol. (2013) 12:48397. doi: 10.1016/S1474-4422(13)70060-7

32. Laatu S, Karrasch M, Martikainen K, Marttila R. Apathy is associated with activities of daily living ability in Parkinson's disease. Dement Geriatr Cogn Disord. (2013) 35:249-55. doi: 10.1159/000347129

33. Massimo L, Evans LK. Differentiating subtypes of apathy to improve personcentered care in frontotemporal degeneration. J Gerontol Nurs. (2014) 40:5865. doi: 10.3928/00989134-20140827-01

34. Caga J, Hsieh S, Highton-Williamson E, Zoing MC, Ramsey E, Devenney $\mathrm{E}$, et al. The burden of apathy for caregivers of patients with amyotrophic lateral sclerosis. Amyotroph Lateral Scler Frontotemporal Degener. (2018) 19:599-605. doi: 10.1080/21678421.2018.1497659

35. König A, Aalten P, Verhey F, Bensadoun G, Petit PD, Robert P, et al. A review of current information and communication technologies: can they be used to assess apathy? Int J Geriatr Psychiatry. (2014) 29:34558. doi: 10.1002/gps.4017

Conflict of Interest: The authors declare that the research was conducted in the absence of any commercial or financial relationships that could be construed as a potential conflict of interest.

Publisher's Note: All claims expressed in this article are solely those of the authors and do not necessarily represent those of their affiliated organizations, or those of the publisher, the editors and the reviewers. Any product that may be evaluated in this article, or claim that may be made by its manufacturer, is not guaranteed or endorsed by the publisher.

Copyright (C) 2021 Zhào, Liu, Xia, Xie and Huang. This is an open-access article distributed under the terms of the Creative Commons Attribution License (CC BY). The use, distribution or reproduction in other forums is permitted, provided the original author(s) and the copyright owner(s) are credited and that the original publication in this journal is cited, in accordance with accepted academic practice. No use, distribution or reproduction is permitted which does not comply with these terms. 